\title{
Análisis de decisión multicriterio en programas de tratamiento de sustitución de opiáceos en trastornos por consumo de opiáceos
}

\section{Multicriteria decision analysis in opioid substitution treatment programs in opioid use disorders}

\author{
Julio Bobes*, Francisco Pascual $* *$, Eliazar Sabater***, Joan Colom****, Francisco Ferre*****, \\ Nestor Szerman*******, Miguel Ángel Casado***, Grupo de trabajo MCDA-OUD*******. \\ * Presidente de la Sociedad Española de Psiquiatría, Catedrático de Psiquiatría de la Universidad de Oviedo. ** Presidente de \\ Socidrogalcohol, Coordinador de la Unidad de Conductas Adictivas de Alcoy (Alicante). *** Pharmacoeconomics \& Outcomes \\ Research Iberia (PORIB). **** Subdirector general de Drogodependencias y Director del Programa de Prevención, Control \\ y Atención al VIH, las ETS y las Hepatitis Víricas. Departamento de Salud, Generalitat de Catalunya. ***** Jefe de Servicio \\ de Psiquiatría B del Hospital Gregorio Marañón, Coordinador de la Estrategia de Salud Mental en el Sistema Nacional de \\ Salud. ****** Presidente de la Fundación Patología Dual, Jefe del Servicio de Salud Mental "Retiro" del Hospital Universitario \\ Gregorio Marañón. ******* Grupo de trabajo MCDA-OUD: Aimee Ruiz, Álvaro Crespo, Ana Beltrán, Carlos Pino, Carmen \\ Ripoll, Carmen Gimeno, Celia del Pino, Cesar Pereiro, Francina Fonseca, Javier Ogando, Juan Ramírez, Juan Jesús Ruiz, \\ Manuel Conde, Manuel Martinez, Marisa Dorado.
}

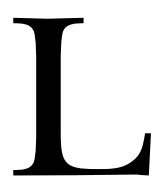
a utilización de nuevas intervenciones sanitarias con un innovador perfil de eficacia y seguridad, pero con mayor impacto presupuestario respecto a tratamientos utilizados anteriormente, se asocia con un aumento de los gastos sanitarios. Por tanto, en la toma de decisiones aparece la necesidad de utilizar criterios adicionales a los tradicionales de evidencia clínica, entre los que destaca la evaluación de la eficiencia, que establece la relación entre los costes y los resultados en salud de las nuevas opciones terapéuticas. En este sentido, los Sistemas Sanitarios basados en el Valor tienen como objetivo fundamental conseguir los mejores resultados en salud para los pacientes, a un coste aceptable y sostenible para estos sistemas. De ello, se deriva que resulte fundamental medir y comparar los resultados en salud de las estrategias utilizadas en una determinada patología (Porter, 2010, McMahon y Chopra, 2012). Sin embargo, hay otros criterios adicionales en la toma de decisiones, como son la relevancia social, prevalencia e incidencia de las enfermedades, el grado de innovación, la efectividad comparada, las necesidades médicas no cubiertas, las decisiones individuales o preferencias de los pacientes, la satisfacción con el tratamiento, el número de pacientes afectados, el empoderamiento de los pacientes y los principios de equidad. Es decir, en muchos casos, considerando el importante número de aspectos a valorar, hay amplias diferencias de criterio en el abordaje de una enfermedad entre los profesionales sanitarios que la tratan, los decisores o gestores políticos que aportan recursos para ese tratamiento y los pacientes que la padecen.

Con el objetivo de facilitar la toma de decisiones, se están incorporando recientemente los análisis de decisión multicriterio (ADMC). Estos análisis suponen una novedad en el abordaje de la toma de decisiones en entornos complejos, ya que permiten sistematizar y simplificar de forma transparente la decisión en diferentes etapas, estableciendo y estimando las preferencias de los decisores e influenciadores (médicos, farmacéuticos, personal de enfermería, psicólogos, gestores y pacientes) de una manera explícita (Thokala et al., 2016, Marsh et al., 2016).

Es sabido que los trastornos por consumo de opiáceos (TCO) representan un problema de salud pública, desde la perspectiva asistencial y social (Torrens, Mestre-Pintó, Montanari, Vicente, J. y Domingo-Salvany, 2017, Pedrero-Pérez y MethaQoL, 2017, Barrio et al., 2017, Martinez-Luna et al., 2018). En Europa, se ha estimado que los consumidores de opiáceos tienen una probabilidad de al menos 5-10 veces mayor de mortalidad que el resto de población de la mis-

Recibido: Febrero 2018; Aceptado: Abril 2018.

Enviar correspondencia a:

Eliazar Sabater. Pharmacoeconomics \& Outcomes Research Iberia (PORIB).

Paseo Joaquín Rodrigo 4-I. 28224 Pozuelo de Alarcón. Teléfono: 9171591 47. E-mail: esabater@porib.com 


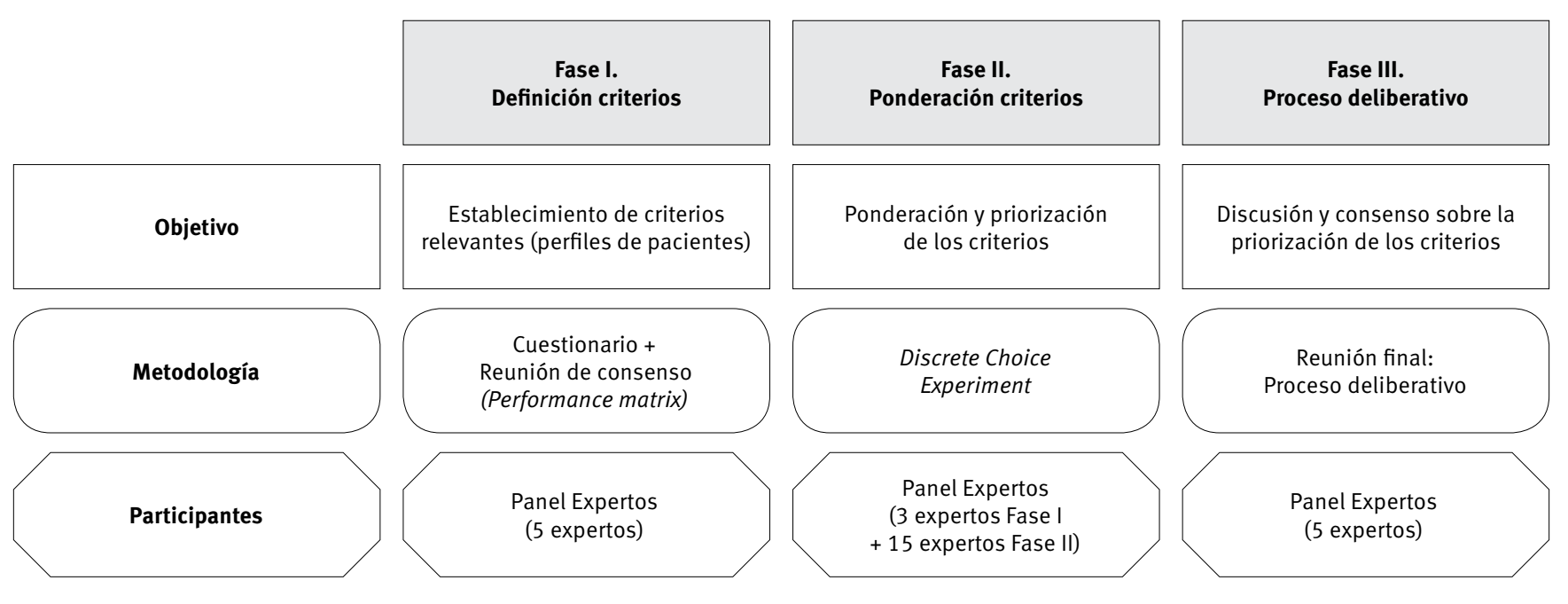

Figura 1. Fases del Análisis de Decisión Multicriterio (ADMC) en programas de tratamiento de sustitución de opiáceos en trastornos por consumo de opiáceos

ma edad y género (Observatorio Europeo de las Drogas y las Toxicomanías, 2017). Asimismo, en Estados Unidos, se ha detectado un aumento de las muertes asociadas a las sobredosis de heroína y opiáceos de laboratorio legales e ilegales (Hedegaard, Warmer y Miniño 2017).

Por todo ello, para aportar información que nos ayude a dimensionar los TCO y facilitar la toma de decisiones sobre su tratamiento, actualmente se está desarrollando un análisis de decisión multicriterio patrocinado por Indivior España. El objetivo de este proyecto es establecer, mediante la metodología del ADMC, cuáles serían los resultados en salud de mayor relevancia y cuál sería su peso en la evaluación de los programas de tratamiento de sustitución de opiáceos en pacientes con TCO.

En este proyecto, con una participación de un Panel de 20 Expertos en el manejo de las adicciones, se han establecido 3 fases, en base a las recomendaciones internacionales de la International Society for Pharmacoeconomics and Outcomes Research (ISPOR) (Thokala et al., 2016; Marsh et al., 2016) (Figura 1): i) una primera fase de definición de las medidas de resultados en salud que se deberían considerar en la evaluación de los pacientes con TCO; ii) una segunda fase de cribado y ponderación donde se estima el peso de cada uno de estos criterios en la toma de decisiones; basado en la metodología de Experimentos de Elecciones Discretas (Discrete Choice Experiments, DCE) y iii) una fase final consistente en un proceso deliberativo, para valorar a nivel global cada uno de los criterios considerados en las fases anteriores.

En una primera reunión con el Panel de Expertos, los criterios considerados para valorar los resultados en salud en los pacientes con TCO en España han sido: consumo de sustancias (opiáceos, alcohol, tabaco, estimulantes y cannabis; según criterios DSM-5), otros trastornos mentales (trastorno afectivo ansioso, psicosis y riesgo de suicidio, trastorno por déficit de atención e hiperactividad, trastorno límite de personalidad, trastornos de personalidad antiso- cial, trastorno por juego y otras alteraciones del control de los impulsos; según criterios DSM-5), nivel de discapacidad (mediante el cuestionario WHODAS II), adherencia, enfermedades médicas (comorbilidades médicas, conductas de riesgo, enfermedades infecciosas y de transmisión sexual), aspectos psicosociales (conducta hostil y/o violenta, presencia de problemas laborales), discapacidad funcional (calidad de vida -a través del cuestionario SF-36-, satisfacción con el tratamiento y con el servicio -según Escala de Verona de Satisfacción con el Servicio-, funcionalidad social -mediante Escala de Apoyo Social de Duke-UNC).

Además, el Panel de Expertos consideró que había determinados factores relacionados con el perfil de los pacientes que podían influir en la valoración de dichos criterios: segmento de edad (menores de 18 años, de 18 a 24 años, de 25 a 34 años, de 35 a 44 años y mayores de 45), recaídas (sí o no), periodo de tiempo con adicción (menor de un año, de 1 a 2 años y mayor de 2 años), número de tratamientos previos recibidos (ninguno, uno, dos, tres o más), tipo de tratamientos previos recibidos (antagonistas opiáceos, agonistas opiáceos y tratamiento libre de fármacos), localización donde se recibieron los tratamientos previos (régimen ambulatorio, centro de día y unidad de deshabituación residencial), antecedentes delictivos (encausado judicialmente por delitos relacionados con el consumo de sustancias y no encausado).

El número de ADMC a nivel internacional es escaso y se ha centrado en patologías concretas, como enfermedades raras o VIH/SIDA (Paulden, Stafinski, Menon, y McCabe 2015, Radaelly, Lettieri y Masella 2014, Sussex et al., 2013, Youngkon, Teerawattananon, Tantivess, y Baltussen, 2012). Sin embargo, ya se han realizado este tipo de análisis para la evaluación de medicamentos huérfanos en Cataluña (Gilabert-Perramon et al., 2017). Además, en la actualidad existe experiencia de la aplicación de los ADMC en el campo de las adicciones, en Reino Unido, con el objetivo de ponderar 
el daño derivado del consumo de drogas en el usuario y otros individuos. Además, los ADMC podrían ser una herramienta que facilitase la implantación de nuevas formas de financiación como el pago por resultados. En este sentido, ya se han producido iniciativas promovidas por el Gobierno de Reino Unido, el cuál realizó una propuesta para medir los resultados en el tratamiento de las adicciones y establecer cómo los acuerdos de pago por resultados podrían utilizarse para sufragar los servicios de tratamiento de adicciones (UK Goverment Policy Paper, 2013). Por todo ello, los ADMC pueden convertirse en una metodología que favorezca la toma de decisiones y la gestión socio-sanitaria de los recursos existentes, incorporando información sobre resultados en salud, evidencia farmacoeconómica y criterios éticos, incorporando en la selección de intervenciones sanitarias a todos los agentes decisores, desde una perspectiva multidisciplinar, en patologías como los TCO.

\section{Conflicto de intereses}

Para el desarrollo de este proyecto, Pharmacoeconomics \& Outcomes Research Iberia (PORIB), una consultora independiente especializada en Evaluación de Intervenciones Sanitarias, ha recibido financiación no condicionada a resultados, por parte de Indivior España.

\section{Referencias}

Barrio, P., Ezzeldin, M., Bruguera, P., Pérez, A., Mansilla, S., Fàbrega, M.,... Balcells, M. (2016). Metadona para el tratamiento de la dependencia de opioides de prescripción médica. Una revisión retrospectiva de historias clínicas. Adicciones, 29, 55-60. doi:10.20882/adicciones.832.

Gilabert-Perramon, A., Torrent-Farnell, J., Catalan, A., Prat, A., Fontanet, M., Puig-Peiró, R.,... M., Badia, X. (2017). Drug evaluation and decision making in Catalonia: development and validation of a methodological framework based on Multi-Criteria Decision Analysis (MCDA) for orphan drugs. International Journal of Technology Assessment in Health Care, 33, 111-120. doi:10.1017/ S0266462317000149.

Hedegaard, H., Warner, M. y Miniño, A. M. (2017). Drug overdose deaths in the United States, 1999-2015. National Center for Health Statistics Data Brief, 273, 1-8.

Marsh, K., IJzerman, M., Thokala, P., Baltussen, R., Boysen, M., Kaló, Z.,... ISPOR Task Force (2016). Multiple Criteria Decision Analysis for Health Care Decision Making-Emerging Good Practices: Report 2 of the ISPOR MCDA Emerging Good Practices Task Force. Value in Health, 19, 125-137. doi:10.1016/j.jval.2015.12.016.

Martínez-Luna, N. G., Rodríguez-Cintas, L., Esojo, A., Palma-Álvarez, R. F., Robles-Martínez, M., Grau-López, L.,... Roncero, C. (2018). Harm reduction program use, psychopathology and medical severity in patients with methadone maintenance treatment. Adicciones, 30, 197207. doi:10.20882/adicciones.897.

McMahon, L.F. y Chopra, V. (2012). Health care cost and value: the way forward. Journal of the American Medical Association, 307, 671-672. doi:10.1001/jama.2012.136.

Nutt, D. J., King, L. A. y Phillips, L. D. (2010). Independent Scientific Committee on Drugs. Drug harms in the UK: a multicriteria decision analysis. Lancet, 376, 1558-1565. doi:10.1016/S0140-6736(10)61462-6.

Observatorio Europeo de las Drogas y las Toxicomanías (2017). Informe Europeo sobre Drogas 2017: Tendencias y novedades. Oficina de Publicaciones de la Unión Europea, Luxemburgo. Recuperado de http://www. emcdda.europa.eu/system/files/publications/4541/ TDAT17001ESN.pdf.

Paulden, M., Stafinski, T., Menon, D. y McCabe, C. (2015). Value-based reimbursement decisions for orphan drugs: A scoping review and decision framework. Pharmacoeconomics, 33, 255-269. doi:10.1007/s40273-014-0235-x.

Pedrero-Pérez, E. y MethaQoL, G. (2016). Dosis de metadona y su relación con calidad de vida, satisfacción, psicopatología, rendimiento cognitivo y consumo adicional de sustancias no prescritas. Adicciones, 29, 37-54. doi:10.20882/adicciones.831.

Radaelly, G., Lettieri, E. y Masella, C. (2014). Implementation of Eunetha core Model in Lombardia: The VTS framework. International Journal of Technology Assessment in Health Care, 30, 105-112. doi:10.1017/ S0266462313000639.

Sussex, J., Rollet, P., Garau, M., Schmitt, C., Kent, A. y Hutchings, A. (2013). A pilot study of multicriteria decision analysis for valuing orphan medicines. Value in Health, 16, 1163-1169. doi:10.1016/j.jval.2013.10.002.

Thokala, P., Devlin, N., Marsh, K., Baltussen, R., Boysen, M.,... Ijzerman, M. (2016). Multiple Criteria Decision Analysis for Health Care Decision Making-An Introduction: Report 1 of the ISPOR MCDA Emerging Good Practices Task Force. Value in Health, 19, 1-13. doi:10.1016/j.jval.2015.12.003.

Torrens, M., Mestre-Pintó, J. I., Montanari, L., Vicente, J. y Domingo-Salvany, A. (2017). Dual diagnosis: An European perspective. Adicciones, 29, 3-5. doi:10.20882/ adicciones.933.

United Kingdom Goverment (2013). Payment by Results drugs recovery pilot: draft outcome definitions. United Kingdom Goverment Department of Health and Social Care. Recuperado de https://www.gov.uk/government/publications / piloting-payment-by-results-for-drugs-recovery-draft-outcome-definitions.

Youngkong, S., Teerawattananon, Y., Tantivess, S. y Baltussen, R. (2012). Multi-criteria decision analysis for setting priorities on HIV/AIDS interventions in Thailand. Health Research Policy and Systems, 10, 6. doi:10.1186/1478 4505-10-6. 